\title{
Proton decay of high-lying states in odd nuclei
}

\author{
Ch. Stoyanov \\ Institute for Nuclear Research and Nuclear Energy, \\ boul. Tzarigradsko Chaussee 72, 1784 Sofia, Bulgaria \\ V. V. Voronov \\ Bogoliubov Laboratory of Theoretical Physics, \\ Joint Institute for Nuclear Research, \\ 141980 Dubna, Moscow Region, Russia \\ N. Van Giai \\ Groupe de Physique Théorique, Institut de Physique Nucléaire, \\ F-91406 Orsay Cedex, France
}

(November 1999)

\begin{abstract}
In the framework of the quasiparticle-phonon model, we study the nonstatistical proton decay of excited states in odd nuclei towards low-lying collective states. Partial cross sections and branching ratios for the proton decay of the high angular momentum states in ${ }^{41} \mathrm{Sc},{ }^{59} \mathrm{Cu}$ and ${ }^{91} \mathrm{Nb}$ are evaluated. The calculated branching ratios predict strong direct proton decays to the low-lying vibrational states in ${ }^{41} \mathrm{Sc}$ and ${ }^{91} \mathrm{Nb}$. A general agreement with existing experimental data is found.
\end{abstract}

Typeset using REVTEX 


\section{INTRODUCTION}

Nucleon transfer reactions induced by hadronic probes at intermediate energies favour the excitation of high angular momentum states lying above particle emission threshold [1 15$]$. The observed structures originate from the coupling of the initial single-particle mode with more complex states. The coupling of single-particle states with surface vibrations is mainly responsible for the damping of the single-particle mode [1,6, 7]. The particle decay of highly excited states gives the opportunity to study in detail the damping process. For example, the relative contributions of the direct and statistical components to the damping of singleparticle mode can be found. Up to now, experimental data and theoretical calculations are available mainly for the neutron decay of high-lying single-particle modes. Very recently, the proton decays of high-lying states in ${ }^{41} \mathrm{Sc},{ }^{59} \mathrm{Cu}$ and ${ }^{91} \mathrm{Nb}$ have been measured [8,9].

In odd nuclei, the simplest excited states can be described as admixtures of singleparticle states or quasiparticle states coupled to collective excitations (or phonons) of the even-even core. This weak coupling picture has been successfully applied to obtain the strength functions of a variety of odd nuclei [1,6]. A method for calculating particle escape widths in the framework of the quasiparticle-phonon model (QPM) [0, 10] has been suggested in Ref. [6] where the inclusive semi-direct neutron decays of high-lying states in ${ }^{209} \mathrm{~Pb}$ have been studied using the general procedure of Refs. [11] [13] which was established for the case of nucleon emission from giant resonances. In a previous work [14 we have extended the method of Ref. [6] in order to calculate non-statistical particle decays of excited states in odd nuclei leading to exclusive channels which correspond to the ground and low-lying excited states of the even-even core. In the present work we apply our method to study the proton decay. Using the QPM we calculate the partial cross sections and branching ratios for the proton decay of the high angular momentum states in ${ }^{41} \mathrm{Sc},{ }^{59} \mathrm{Cu}$ and ${ }^{91} \mathrm{Nb}$ and compare them with experimental data.

This paper is organized as follows: in Sec. II we describe briefly our theoretical approach to treat the direct nucleon decay of high angular momentum states of single-particle type. In Sec. III a comparison of the calculated and measured branching ratios for non-statistical proton decay of high-lying states in ${ }^{41} \mathrm{Sc},{ }^{59} \mathrm{Cu}$ and ${ }^{91} \mathrm{Nb}$ is presented. Finally, in Sec. IV conclusions are drawn.

\section{THEORY}

\section{A. The projection operator method}

The projection operator method is a convenient approach to treat problems involving single-particle continua in a many-body context. The general formalism was introduced by Yoshida and Adachi [11] and it has been applied to studies of high-lying states in odd nuclei [6.14]. The detailed expressions can be found in Ref. [14]. Here, we simply recall the main features.

We write the hamiltonian of the $\mathrm{A}+1$ system in the form:

$$
H=h+H_{\text {core }}+H_{\text {coupl }} .
$$


The first term describes the motion of a particle in an average potential $U$ created by the particles in the core:

$$
h=-\frac{1}{2 m} \nabla^{2}+U
$$

The core hamiltonian is a sum of single-particle hamiltonians $h_{i}$ and two-body residual interactions $V_{i, j}$ :

$$
H_{\text {core }}=\sum_{i=1}^{A} h_{i}+\sum_{i<j}^{A} V_{i, j} .
$$

The last term of $H$ is a sum of interactions between the odd particle and the core particles:

$$
H_{\text {coupl }}=\sum_{i=1}^{A} V_{0, i} .
$$

The physical spectrum of $h$ consists of a small number of bound states $\left\{\varphi_{i}, e_{i}\right\}$ and a continuum of scattering states $\left\{\varphi_{e}, e\right\}$ which form altogether a complete, orthogonal basis. The projection operator method consists in introducing another complete set of orthogonal basis states which is a direct sum of two complementary subsets, a first subset of discrete, orthonormal states $\left\{\phi_{\alpha}, \epsilon_{\alpha}\right\}$ which span the single-particle $q$ space and a second subset of $\left\{\phi_{\epsilon}, \epsilon\right\}$ continuum states spanning the complementary $p \equiv 1-q$ space. We will denote by $a_{\alpha}^{\dagger}, a_{\alpha}\left(a_{\epsilon}^{\dagger}, a_{\epsilon}\right)$ the creation and annihilation operators of state $\varphi_{\alpha}\left(\varphi_{\epsilon}\right)$.

In the spirit of the QPM [7] the hamiltonian $H_{\text {core }}$ is treated in the random-phase approximation (RPA) in a discrete space, i.e., the particle-hole configurations of RPA are built only with q-space states. We denote by $E_{\nu}$ and $O_{\nu}^{\dagger}$ the energies and creation operators of these RPA states which describe core excitations. If $\mid 0>$ represents the RPA ground state of the core, the properties of the $(\mathrm{A}+1)$-nucleus except for its nucleon decay properties can be described in terms of the one-particle states $a_{\alpha}^{\dagger} \mid 0>$ and one-particle-plus-phonon states $\left[a_{\beta}^{\dagger} \otimes O_{\nu}^{\dagger}\right] \mid 0>$. We can write:

$$
\begin{aligned}
\mid d_{i}> & \equiv d_{i}^{\dagger} \mid 0> \\
& =\left(\sum_{\alpha} C_{\alpha}^{(i)} a_{\alpha}^{\dagger}+\sum_{\beta, \nu} D_{\beta, \nu}^{(i)}\left[a_{\beta}^{\dagger} \otimes O_{\nu}^{\dagger}\right]\right) \mid 0>
\end{aligned}
$$

We call Q space the space spanned by the (real) state vectors $\mid d_{i}>$ and $Q$ the corresponding projection operator. The amplitudes $C_{\alpha}^{(i)}$ and $D_{\beta, \nu}^{(i)}$, and the energies $\omega_{i}$ of $\mid d_{i}>$ are determined by diagonalizing $H$ in the RPA, i.e., one solves:

$$
\left[Q H Q, d_{i}^{\dagger}\right]=\omega_{i} d_{i}^{\dagger}
$$

within the approximation of commutator linearization. The distribution of $\left|C^{(i)}\right|^{2}$ represents the strength function from which one can deduce the spectroscopic factors [1].

To allow for nucleons to decay it is necessary to introduce state vectors where the odd particle has a non-zero probability of being at infinity. This is achieved by constructing the $\mathrm{P}$ space complementary to Q space and consisting of all states which are linear combinations of the following one-particle and one-particle-plus-phonon configurations: 


$$
\left|\epsilon>\equiv a_{\epsilon}^{\dagger}\right| 0>, \quad\left|\epsilon, \nu>\equiv\left[a_{\epsilon}^{\dagger} \otimes O_{\nu}^{\dagger}\right]\right| 0>.
$$

The present definition of $\mathrm{P}$ space neglects continuum effects on the phonons $O_{\nu}^{\dagger}$ which can also in principle couple to the continuum and emit nucleons by themselves. Actually, these effects should have a small influence on the non-statistical particle decay of the $(A+1)$ nucleus since the most important phonons contributing to the particle-phonon coupling are the low-lying collective states of the core.

The direct sum of $\mathrm{Q}$ space and $\mathrm{P}$ space is by construction the complete particle-plusphonon space in which the hamiltonian $H$ should be solved. It is completely equivalent to solve in the simpler $\mathrm{Q}$ space the more complicated effective hamiltonian:

$$
\begin{aligned}
\mathcal{H}(E) & \equiv Q H Q+Q H P \frac{1}{E^{(+)}-P H P} P H Q \\
& \equiv H_{Q Q}+W(E),
\end{aligned}
$$

where $P$ is the projection operator onto $\mathrm{P}$ space $(P+Q=1)$ and $E$ is the energy of the system. The hamiltonian $\mathcal{H}$ is complex and energy dependent. For each value of $E$ one has to find the set of complex states and eigenenergies:

$$
\begin{aligned}
\left|\mathcal{D}_{i}\right\rangle & \equiv \mathcal{D}_{i}^{\dagger}|0\rangle, \\
\Omega_{i} & \equiv \bar{\omega}_{i}-\frac{i}{2} \Gamma_{i}^{\uparrow},
\end{aligned}
$$

satisfying:

$$
\left[\mathcal{H}(E), \mathcal{D}_{i}^{\dagger}\right]=\Omega_{i} \mathcal{D}_{i}^{\dagger}
$$

\section{B. Escape widths}

We consider a direct transfer reaction $a+A \rightarrow b+(A+1)^{*}$ followed by a sequential decay $(A+1)^{*} \rightarrow p+A^{*}$ where the $(\mathrm{A}+1)$-nucleus in a highly excited state decays by a semi-direct proton emission. In this process, the $\mid \mathcal{D}_{i}>$ states will act as doorway states. If we describe the reaction mechanism in a simple approach, e.g., a distorted wave Born approximation (DWBA), we can write down the scattering amplitude from an initial channel $i$ where the target $A$ is in its ground state $\mid 0>$ to a final channel $f$ where the residual nucleus is left in some excited state $\left|\nu>=O_{\nu}^{\dagger}\right| 0>$ with excitation energy $E_{\nu}$ while the escaping proton has an energy $E-E_{\nu}$. Using the complex bi-orthogonal basis $\left\{\left|\mathcal{D}_{i}>,<\overline{\mathcal{D}}_{i}\right|\right\}$, we have:

$$
T_{f i}=\sum_{l j} \sum_{i} \frac{<\phi_{l j}^{(-)}\left(E-E_{\nu}\right), \nu|H| \mathcal{D}_{i}><\overline{\mathcal{D}}_{i}, b|V| 0, a>}{E-\bar{\omega}_{i}+i \Gamma_{i}^{\uparrow} / 2},
$$

where $V$ is the interaction inducing the particle transfer from $a$ to $A, \phi^{(-)}$is an incoming wave of $p$ space at energy $E-E_{\nu}$, and the sum over $(l, j)$ is restricted by the angular momenta and parities of states $\left|\mathcal{D}_{i}\right\rangle$ and $\nu$. The case where the final channel is the ground state of the residual nucleus corresponds to the above expression with $\nu=0, E_{\nu}=0$. 
The nucleon-transfer matrix element $\left\langle\overline{\mathcal{D}}_{i}, b|V| 0, a>\right.$ is proportional to the onequasiparticle amplitude of the state $\left|\mathcal{D}_{i}\right\rangle$. In analogy with the amplitude $C^{(i)}$ of Eq.(5), we denote it by $\mathcal{C}^{(i)}$. We also introduce the partial escape amplitudes of state $\mid \mathcal{D}_{i}>$ to channel $\nu$ :

$$
\gamma_{i, \nu}(l j) \equiv \sqrt{2 \pi}<\phi_{l j}^{(-)}\left(E-E_{\nu}\right), \nu|H| \mathcal{D}_{i}>
$$

The partial widths are:

$$
\Gamma_{i, \nu}^{\uparrow} \equiv \sum_{l j}\left|\gamma_{i, \nu}(l j)\right|^{2}
$$

To obtain an expression for the cross section simple enough to lend itself to a discussion in terms of escape widths, let us assume furthermore that interference terms between different doorway states can be neglected. The density of $\left|\mathcal{D}_{i}\right\rangle$ states is large, and for each interval centered around $E$ and containing $N$ states we define locally averaged quantities:

$$
\begin{aligned}
C^{2}(E) & \equiv \sum_{i \in I}\left|C^{(i)}\right|^{2} / N \\
\Gamma_{\nu}^{\uparrow}(E) & \equiv \sum_{i \in I} \Gamma_{i, \nu}^{\uparrow} / N \\
\Gamma^{\uparrow}(E) & \equiv \sum_{\nu} \Gamma_{\nu}^{\uparrow}(E)
\end{aligned}
$$

Then, one can rewrite the cross sections in the following form:

$$
\sigma_{\nu}(E) \propto C^{2}(E) \Gamma_{\nu}^{\uparrow}(E) / \Gamma^{\uparrow}(E)
$$

The branching ratios can be calculated by the following formula:

$$
B_{\nu}=\frac{\sigma_{\nu}(E)}{\sum_{\mu} \sigma_{\mu}(E)}=\frac{\Gamma_{\nu}^{\uparrow}(E)}{\Gamma^{\uparrow}(E)} .
$$

\section{Inputs of the model}

The above formalism is applied to study the semi-direct proton decay of the nuclei ${ }^{41} \mathrm{Sc}$, ${ }^{59} \mathrm{Cu}$ and ${ }^{91} \mathrm{Nb}$ with the aim of comparing the predictions with existing data from exclusive measurements.

Although the QPM model Soloviev et al. [7, 10] is not fully consistent since the residual interaction between quasiparticles is not derived from the quasiparticle mean field, it has the advantage that its two-body residual interaction is chosen of a multipole-multipole separable form and therefore, it enables one to work with configuration spaces of large dimensions without facing the cumbersome problem of diagonalizing large matrices. This is very important for the results to be meaningful as it was already shown in a previous work on neutron emission [14.

The mean potentials entering the single-particle hamiltonians $h_{i}$ are of Woods-Saxon form. Their parameters are chosen according to Refs. 15,16 with some readjustments 
such that, once the coupling to phonons is included, the ground states are located at their respective experimental positions with respect to the proton separation threshold. The QPM hamiltonian includes the monopole pairing which must be taken into account for nuclei with open shells [1]. This is the case when we calculate the core properties of ${ }^{59} \mathrm{Cu}$ and ${ }^{91} \mathrm{Nb}$.

The residual particle-hole interaction of Eq. (3) is taken of a separable form in coordinate space with effective interaction strengths considered as adjustable parameters. For the radial interaction form factor we have used $f(r)=d U / d r$ where $U(r)$ is the central part of the Woods-Saxon potential. For each excitation mode of the even-even core corresponding to a given angular momentum, parity and isospin, the interaction strength is found by requiring that the lowest collective state calculated in RPA be at the experimental energy. These interaction strengths are also used for the quasiparticle-phonon coupling. We have modified the single-particle proton spectrum for ${ }^{91} \mathrm{Nb}$ in comparison with Ref. [14] to get a better description of high-lying $1 g_{7 / 2}, 1 h_{11 / 2}$ subshells, but so far as energies of levels near the Fermi surface are practically the same there are no changes in the properties of low-lying vibrational states in comparison with those of Ref. [14]. For example, the calculated $\mathrm{B}(\mathrm{E} \lambda)$ values of the first low-lying collective states $3_{1}^{-}, 5_{1}^{-}$in ${ }^{40} \mathrm{Ca}$ are $\mathrm{B}(\mathrm{E} 3)=1.36 e^{2} \mathrm{fm}^{6}, \mathrm{~B}(\mathrm{E} 5)=2.67 e^{2} \mathrm{fm}^{10}$ that can be compared with the corresponding experimental values $1.24 e^{2} \mathrm{fm}^{6}$ and $2.97 e^{2} \mathrm{fm}^{10}$. For all nuclei under consideration the calculated values of $\mathrm{B}(\mathrm{E} \lambda)$ are in general agreement with experiment. In the present model, these low-lying phonons of the core are the physical channels where the initial state in the excited odd-A nucleus can decay by semi-direct proton emission. In the actual calculation, a very large number of RPA phonons are included in the quasiparticle-phonon basis, but for ${ }^{40} \mathrm{Ca}$ the first quadrupole state cannot be treated as a one-phonon or particle-hole state and it is outside the present model. On the other hand, the experimental data [17] show that the proton decay of ${ }^{41} \mathrm{Sc}$ to the $2_{1}^{+}$state in ${ }^{40} \mathrm{Ca}$ is very weak.

At high excitation energies, when the level density becomes large, it is often more convenient to calculate the strength function [18] instead of solving the secular equations (10). One defines the strength function $\bar{C}_{\alpha}^{2}(E)$ as the strength distribution $\left|C_{\alpha}^{(i)}\right|^{2}$ folded with an averaging function $\rho$ :

$$
\bar{C}_{\alpha}^{2}(E)=\sum_{i}\left|C_{\alpha}^{(i)}\right|^{2} \rho\left(E-\Omega_{i}\right)
$$

where $\rho(z)$ is usually chosen as a Breit-Wigner function, $\rho(z)=\frac{\Delta}{2 \pi} \frac{1}{z^{2}+\Delta^{2} / 4}$. It is shown in Ref. [14] that the calculation of the strength function $\bar{C}_{\alpha}^{2}(E)$ can be done without the detailed knowledge of the amplitudes $\left|C_{\alpha}^{(i)}\right|^{2}$. The same averaging procedure can be applied to calculate the partial escape widths and the branching ratios. In all calculations performed in this work we have adopted the value $\Delta=0.1 \mathrm{MeV}$.

\section{RESULTS AND DISCUSSION}

Using the methods presented above we have calculated the cross sections and branching ratios for the non-statistical proton decay of high-lying states with high angular momenta in ${ }^{41} \mathrm{Sc},{ }^{59} \mathrm{Cu}$ and ${ }^{91} \mathrm{Nb}$ going to the ground and low-lying collective states of ${ }^{40} \mathrm{Ca},{ }^{58} \mathrm{Ni}$ and ${ }^{90} \mathrm{Zr}$, respectively. 
An example of cross section calculations by the strength function method is shown in Fig. 1 for the case of proton decay of the $g_{9 / 2}$ states in ${ }^{41} \mathrm{Sc}$. Since the calculated cross section is proportional to the square of the one quasiparticle amplitude of the wave function this figure enables one to see the $1 g_{9 / 2}$ strength distribution in ${ }^{41} \mathrm{Sc}$, too. The $1 g_{9 / 2}$ strength is distributed over a broad energy interval due to the coupling with the collective vibrations. Results of our calculations for the $1 g_{9 / 2}$ proton particle strength and experimental data obtained from the ${ }^{40} \mathrm{Ca}\left({ }^{3} \mathrm{He}, \mathrm{dp}\right)$ reaction at $E_{3} \mathrm{He}=240 \mathrm{MeV}$ [17] are presented in Table 1. It is seen from this table that the calculations reproduce reasonably well the integral characteristics of the $1 g_{9 / 2}$ proton strength distribution in ${ }^{41} \mathrm{Sc}$.

The partial contributions of the non-statistical proton decay into the ground state and $3_{1}^{-}, 5_{1}^{-}$states of ${ }^{40} \mathrm{Ca}$ are presented in Fig. 2. It is seen from Fig. 2 that the partial cross sections are very energy dependent and as a result different channels can dominate at some excitation energies. The solid curve in Fig. 1 presents the sum of all 3 partial channels. As one can see from Fig. 2 at excitation energies below $8.4 \mathrm{MeV}$ the ground state channel dominates, but there are strong transitions to the $5_{1}^{-}$state at 7.5 and $8.0 \mathrm{MeV}$. The $3_{1}^{-}$and $5_{1}^{-}$channels begin to contribute importantly starting from $8.4 \mathrm{MeV}$ and they become the main contributors after $9.5 \mathrm{MeV}$.

The sum of partial widths for different channels in some energy intervals are given in Table 2. As one can see from Table 2 for the energy interval $2.0-12.4 \mathrm{MeV}$ the proton decay to the $5_{1}^{-}$state gives a contribution of about $54 \%$ and the $3_{1}^{-}$channel contributes about $37 \%$ in the total sum. There is a predominance of the $5_{1}^{-}$channel in comparison with the $3_{1}^{-}$one in the energy interval 7.0 - 12.4 MeV. Such a behaviour can be understood from the structure of the decaying states and the angular momentum and energy carried away by the emitted proton. It is seen from Eq. (12) that the partial escape amplitude for fixed values of $l j$ is proportional to the contribution of the quasiparticle-plus-phonon configuration made of a quasiparticle with the same $l j$ and a phonon of the final state. The cross section depends on the square of these amplitudes summed over all $l j$ allowed by the angular momentum coupling rules. In the case of the proton decay of the $g_{9 / 2}$ states at excitation energies $7-12 \mathrm{MeV}$ into the $3_{1}^{-}$channel the outgoing protons carry $(l, j)=(1,3 / 2)$ mainly whereas it is $(l, j)=(1,3 / 2)$ and $(l, j)=(1,1 / 2)$ for the $5^{-}$channel. Thus, penetration factor arguments favour the latter channel. It is well known that the strongest coupling between the single-particle states and phonons takes place for the collective phonons. That is why the coupling with the low-lying vibrations is mainly responsible of the damping of the high-lying single-particle modes and giant resonances in nuclei. This is the case for the $3_{1}^{-}$and $5_{1}^{-}$states in ${ }^{40} \mathrm{Ca}$. By this reason the strengths of the $3_{1}^{-}$and $5_{1}^{-}$channels are redistributed in a broad energy interval. The decrease of the spectroscopic strength with an increase of the excitation energy leads to a decreasing of cross sections of the channels discussed above. It is worth to mention that a very similar behaviour for the $3_{1}^{-}$and $5_{1}^{-}$ channels for the neutron decay of the high angular momentum states in ${ }^{209} \mathrm{~Pb}$ also takes place [14. Our conclusions about the role of different channels for the proton decay in ${ }^{41} \mathrm{Sc}$ agree with observations made in Ref. [17], but no separation of the direct part from the statistical one for the proton decay was done in that work.

As a second example we consider the proton decay of the $g_{9 / 2}$ states in ${ }^{59} \mathrm{Cu}$. Results of our calculations for the $g_{9 / 2}$ strength distribution in ${ }^{59} \mathrm{Cu}$ and experimental data [19] are presented in Table 3. The calculations reproduce correctly the strength near $7 \mathrm{MeV}$ and 
this part is mainly responsible for the proton decay. For the excitation energy interval (2 - 8.9) $\mathrm{MeV}$ in ${ }^{59} \mathrm{Cu}$ the summed ground state width is equal to $10.3 \mathrm{eV}$ and this is much less than in the ${ }^{41} \mathrm{Sc}$ case. An additional contribution from the $2^{+}$channel has a summed width that is six times less than that of the ground state channel. There are no transitions to other vibrational states. This is easily understood if one looks at the structure of the states under discussion. For the $2^{+}$channel the outgoing protons carry $(l, j)=(4,9 / 2)$ and there is a suppression of such transitions due to penetration factor effects. The configurations including $3_{1}^{-}$and $5_{1}^{-}$states coupled with continuum are located at somewhat higher energies. This is why no proton decay can proceed to the $3_{1}^{-}$and $5_{1}^{-}$states in ${ }^{59} \mathrm{Ni}$.

Very recently, detailed experimental information about proton decay of isobaric analog states (IAS) in ${ }^{91} \mathrm{Nb}$ has been shown in Ref. [9]. The IAS are strongly excited by means of the ${ }^{90} \operatorname{Zr}(\alpha, t)^{91} \mathrm{Nb}$ reaction. A sharp peak is seen at $12 \mathrm{MeV}$ excitation energy. Around this excitation energy three IAS have been observed earlier 20] at $11.93 \mathrm{MeV}, 12.07 \mathrm{MeV}$ and $12.15 \mathrm{MeV}$. It is shown in Ref. 9] that at $12 \mathrm{MeV}$ by means of $(\alpha, t)$ reaction predominantly the $h_{11 / 2}$ state is excited. The proton decays to the ground state and some low-lying states are observed. The partial differential cross sections for each final state are given. The data reveal proton decays predominantly to $5_{1}^{-}$and $3_{1}^{-}$excited states of ${ }^{90} \mathrm{Zr}$.

Experimental data 20] for energies and spectroscopic factors of IAS and results of our calculations are given in Table 4 . Besides, this table also contains calculated partial widths for the proton decay of three IAS to the ground, $2_{1}^{+}, 3_{1}^{-}, 4_{1}^{+}$and $5_{1}^{-}$states.

As one can see from Table 4 the calculated excitation energies for the $7 / 2^{+}$IAS are higher than the experimental ones but the spectroscopic factors extracted from experimental data within $20 \%$ accuracy are reproduced reasonably well.

Let us discuss the proton partial widths for these IAS. It is seen from Table 4 that for the first $7 / 2^{+}$state the main decay channel is the $4_{1}^{+}$channel. For this state the $(l, j)=(0,1 / 2)$ protons are most important for the $4_{1}^{+}$channel and there is a rather weak transition for the $2_{1}^{+}$channel due to the $(l, j)=(2,3 / 2)$ protons. The ground state width is proportional to the one quasiparticle strength (the spectroscopic factor). Since the contribution of the one quasiparticle component in the norm of the wave function of this IAS is much less than contributions of the quasiparticle-plus-phonon components the transition to the ground state is weaker than to the $4_{1}^{+}$. The particularities of the partial proton widths for the second $7 / 2^{+}$state can be understood from the structure of this state, too. For the $4_{1}^{+}$channel the $(l, j)=(2,3 / 2),(2,5 / 2)$ and $(4,7 / 2)$ protons can contribute besides the $(l, j)=(0,1 / 2)$ protons. As a result the decay width to the $4_{1}^{+}$state increases strongly for the second $7 / 2^{+}$IAS in comparison with that of the first $7 / 2^{+}$state. An increase of the contribution of the configuration constructed from the first quadrupole phonon and $2 d_{3 / 2}$ single-particle state in comparison with their contribution to the structure of the first $7 / 2^{+}$state results in an essential growth of the decay width to the $2_{1}^{+}$channel. The ground state width becomes larger mainly because of the increased spectroscopic factor. The transitions to the $5_{1}^{-}$state can take place due to the outgoing protons with $(l, j)=(5,11 / 2)$ but they are supressed because of penetration factors and a small contribution of relevant components in the wave function structure.

In the case of the $11 / 2^{-}$IAS the $5^{-}$channel dominates and this is due to the outgoing protons carrying $(l, j)=(0,1 / 2),(2,3 / 2)$ and $(2,5 / 2)$. The components containing the $3_{1}^{-}$ phonon have a small contribution in the wave function structure and as a result the width 
for the $3_{1}^{-}$channel is small, too. In spite of unfavourable penetration factor the $2_{1}^{+}$width is 4 times larger than that of the $3_{1}^{-}$channel. This is due to the configuration consisting of the $2_{1}^{-}$phonon coupled with $h_{11 / 2}$ which contributes about $15 \%$ in the norm of the $11 / 2^{-}$IAS.

The comparison of the calculated partial widths with the data of Ref. [9] shows that there is a qualitative agreement. The main channels of the proton decay of $(11 / 2)^{-}$IAS are reproduced in the calculated structure. The dominance of the $5_{1}^{-}$channel is well established and the calculated partial width of $3.3 \mathrm{keV}$ (Table 4) is in agreement with the measured one $(2.9 \mathrm{keV})$. The $3_{1}^{-}$channel is more pronounced than ground state channel but the calculated partial widths for both channels are much less than the measured ones. The calculations indicate a large decay to the $2_{1}^{+}$channel. Such decay is discussed in Ref. [9] but quantitative

estimations for the contribution of the $2_{1}^{+}$channel in the cross section have not been evaluated in that work.

The summed partial widths for the proton decay of the three IAS to the low-lying vibrational states are also presented in Table 4. As one can see from the last row of Table 4 the $4_{1}^{+}$channel exhausts almost $60 \%$ of the total cross section, the $5_{1}^{-}$channel contributes about $26 \%$ and the $2_{1}^{+}$channel gives about $10 \%$ of the total strength. The contribution of the ground state channel for the proton decay of the three IAS in ${ }^{91} \mathrm{Nb}$ is $4 \%$ only.

\section{CONCLUSIONS}

A microscopic approach based on the QPM has been applied to calculate the nonstatistical proton decays of high angular momentum states excited in one-nucleon transfer reactions. Partial cross sections and branching ratios for proton emission from high-lying states in ${ }^{41} \mathrm{Sc},{ }^{59} \mathrm{Ni}$ and ${ }^{91} \mathrm{Nb}$ have been evaluated. The calculated branching ratios demonstrate the existence of strong direct proton decays to the low-lying vibrational states in ${ }^{41} \mathrm{Sc}$ and ${ }^{91} \mathrm{Nb}$ and enables one to understand particularities of the decay to different channels. One can conclude from an analysis of calculated partial cross sections that, for high angular momentum states the non-statistical proton decay is more favourable into the higher angular momentum and lower excitation energy final states when the two following conditions are fulfilled: a strong particle-phonon coupling in that channel and a penetration factor which is not hindered by angular momentum or energy. A similar conclusion was also reached for the neutron decay case [14]. A general agreement with existing experimental data is obtained and the predicted branching ratios for different channels can be used to analyze future experimental data.

\section{ACKNOWLEDGMENTS}

We would like to thank G. Crawley and S. Fortier for fruitful discussions and correspondence. Ch.S. and V.V.V. thank the hospitality of IPN-Orsay where the main part of this work was done. This work is partly supported by IN2P3-JINR agreement and by the Bulgarian Science Foundation (contract No Ph. 801). 


\section{REFERENCES}

[1] S. Galès, Ch. Stoyanov and A.I. Vdovin, Phys. Rep. 166 (1988) 127.

[2] S. Galès, C.P. Massolo, S. Fortier, J.P. Schapira, P. Martin and V. Comparat, Phys. Rev. C 31 (1990) 94.

[3] D. Beaumel et al., Phys. Rev. C49 (1994) 2444.

[4] S. Fortier et al., Physics with Recoil Separators and Detector Arrays, Eds. R.K. Bhowmik and A.K. Sinha (Allied Publishers, New Delhi, 1995) p. 380.

[5] S. Fortier et al., Phys. Rev. C 52 (1995) 2410.

[6] N. Van Giai and Ch. Stoyanov, Phys. Lett. B 272 (1991) 178.

[7] V.G. Soloviev, "Theory of atomic nuclei: quasiparticles and phonons", Institute of Physics Publishing, Bristol and Philadelphia, 1992.

[8] G.H. Yoo et al., submitted to Phys. Rev. C (1999); G.M. Crawley and S. Fortier, private communication.

[9] H. K. T. van der Molen, PhD thesis (Groningen, 1999).

[10] A.I. Vdovin, V.V. Voronov, V.G. Soloviev and Ch. Stoyanov, Sov. J. Part. Nucl. 16 (1985) 245.

[11] S. Yoshida and S. Adachi, Z. Phys. A 325 (1986) 441.

[12] N. Van Giai and Ch. Stoyanov, Phys. Lett. B 252 (1990) 9.

[13] G. Colò, P.F. Bortignon, N. Van Giai, A. Bracco and R.A. Broglia, Phys. Lett. B 276 (1992) 279.

[14] N. Van Giai, Ch. Stoyanov, V.V. Voronov and S. Fortier, Phys. Rev. C53 (1996) 730.

[15] V.A. Chepurnov, Sov.J.Nucl.Phys. 6 (1967) 955.

[16] K. Takeuchi and P.A. Moldauer, Phys. Lett. B 28 (1969) 384.

[17] J. Guillot et al., Phys. Lett. B 258 (1990) 271.

[18] A. Bohr and B.R. Mottelson, Nuclear Structure, Vol. I (Benjamin, New York, 1969).

[19] C.M. Baglin, Nuclear Data Sheets 69 (1993) 733.

[20] G. Finkel et al., Phys. Rev. C 19 (1979) 1782. 


\section{FIGURES}

FIG. 1. Cross sections (arbitrary units) for the excitation and proton decay of $g_{9 / 2}$ states in ${ }^{41} \mathrm{Sc}$ into low-lying states, as a function of excitation energy $E$. The solid line is the sum of ground state, $3_{1}^{-}$and $5_{1}^{-}$channels, the dotted line shows the partial contribution of the ground state channel.

FIG. 2. Partial cross sections (arbitrary units) for the excitation and proton decay of $g_{9 / 2}$ states

in ${ }^{41} \mathrm{Sc}$ as a function of excitation energy $E$. The solid line is the $3_{1}^{-}$channel, the dotted-dashed line is $5_{1}^{-}$channel and the dotted line is the ground state channel. 


\section{TABLES}

TABLE I. The $g_{9 / 2}$ proton particle strength in ${ }^{41} \mathrm{Sc}$.

\begin{tabular}{ccccc}
\hline \hline$\Delta E_{x}(\mathrm{MeV})$ & $\overline{E_{x}}($ exp. $)(\mathrm{MeV})$ & $\overline{E_{x}}($ calc. $)(\mathrm{MeV})$ & $C^{2} S($ exp. $)$ & $C^{2} S($ calc. $)$ \\
\hline $2.0-12.4$ & 8.80 & 8.96 & 0.84 & 0.92 \\
$8.4-12.4$ & 10.4 & 9.49 & 0.56 & 0.76 \\
& 5.04 & 4.65 & 0.12 & 0.05 \\
\hline \hline
\end{tabular}

TABLE II. Summed partial widths (in $\mathrm{MeV}$ ) for different channels for the proton decay in ${ }^{41} \mathrm{Sc}$

\begin{tabular}{|c|c|c|c|}
\hline$\Delta E_{x}(\mathrm{MeV})$ & $\sum \Gamma_{g . s .}^{\uparrow}$ & $\sum \Gamma_{5_{1}^{-}}^{\uparrow}$ & $\sum \Gamma_{3_{1}^{-}}^{\uparrow}$ \\
\hline $2.0-7.2$ & 0.225 & $3.63 \quad 10^{-5}$ & $2.14 \quad 10^{-2}$ \\
\hline $2.0-8.4$ & 3.314 & 0.170 & $2.31 \quad 10^{-2}$ \\
\hline $8.4-12.4$ & 1.689 & 11.36 & 7.832 \\
\hline $2.0-12.4$ & 5.003 & 11.53 & 7.855 \\
\hline
\end{tabular}

TABLE III. The $1 g_{9 / 2}$ proton particle strength in ${ }^{59} \mathrm{Cu}$.

\begin{tabular}{ccc}
\hline \hline$E_{x}(\mathrm{MeV})$ & $C^{2} S($ exp. $)$ & $C^{2} S($ calc. $)$ \\
\hline 3.0 & 0.35 & 0.60 \\
$\sim 7.3$ & 0.35 & 0.35 \\
$3.1-7.2$ & 0.09 & 0.05 \\
\hline \hline
\end{tabular}

TABLE IV. Partial widths (in keV) for different channels for the proton decay in ${ }^{91} \mathrm{Nb}$

\begin{tabular}{|c|c|c|c|c|c|c|c|c|c|}
\hline$E_{x}(\exp ).(\mathrm{MeV})$ & $E_{x}($ calc. $)(\mathrm{MeV})$ & $\mathrm{J}^{\pi}$ & $C^{2} S(\exp )$. & $C^{2} S($ calc. $)$ & $\overline{\Gamma_{g . s .}^{\uparrow}}$ & $\overline{\Gamma_{2}^{\uparrow}}$ & $\overline{\Gamma_{3}^{\uparrow}}$ & $\overline{\Gamma_{4_{1}^{+}}^{\uparrow}}$ & $\sum \Gamma_{5_{1}^{-}}^{\uparrow}$ \\
\hline 11.79 & 12.00 & $7 / 2^{+}$ & 0.11 & 0.18 & 0.156 & 0.019 & 0 & 0.990 & 0.005 \\
\hline 12.07 & 12.15 & $11 / 2^{-}$ & 0.35 & 0.57 & 0.049 & 0.296 & 0.073 & 0.002 & 3.308 \\
\hline \multirow[t]{3}{*}{12.15} & 12.90 & $7 / 2^{+}$ & 0.40 & 0.29 & 0.309 & 1.010 & 0 & 6.738 & 0.027 \\
\hline & & & & $\sum \Gamma^{\uparrow}$ & 0.514 & 1.325 & 0.073 & 7.73 & 3.340 \\
\hline & & & & $\%$ of total $\sum \Gamma^{\uparrow}$ & 4.0 & 10.2 & 0.6 & 59.5 & 25.7 \\
\hline
\end{tabular}




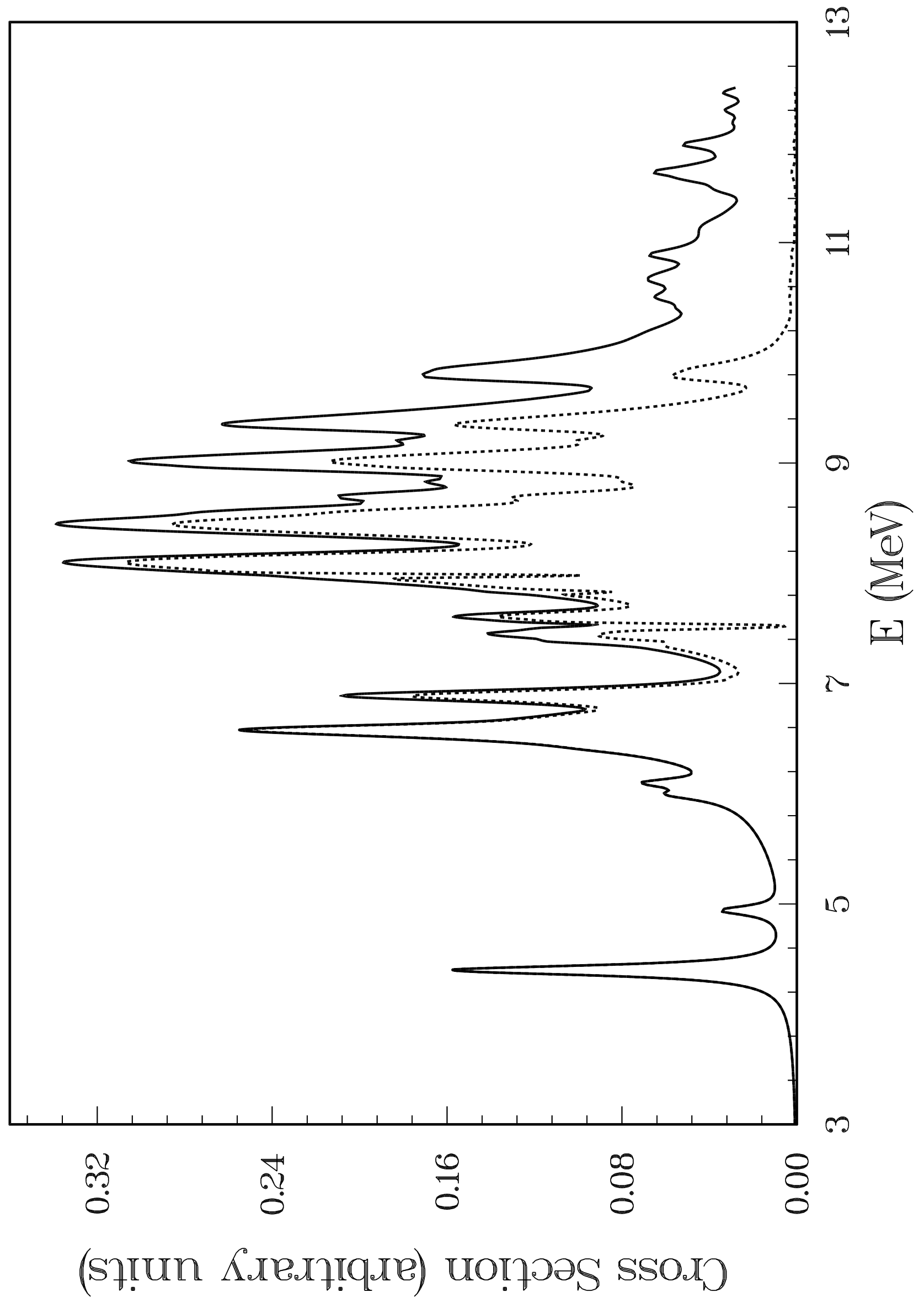




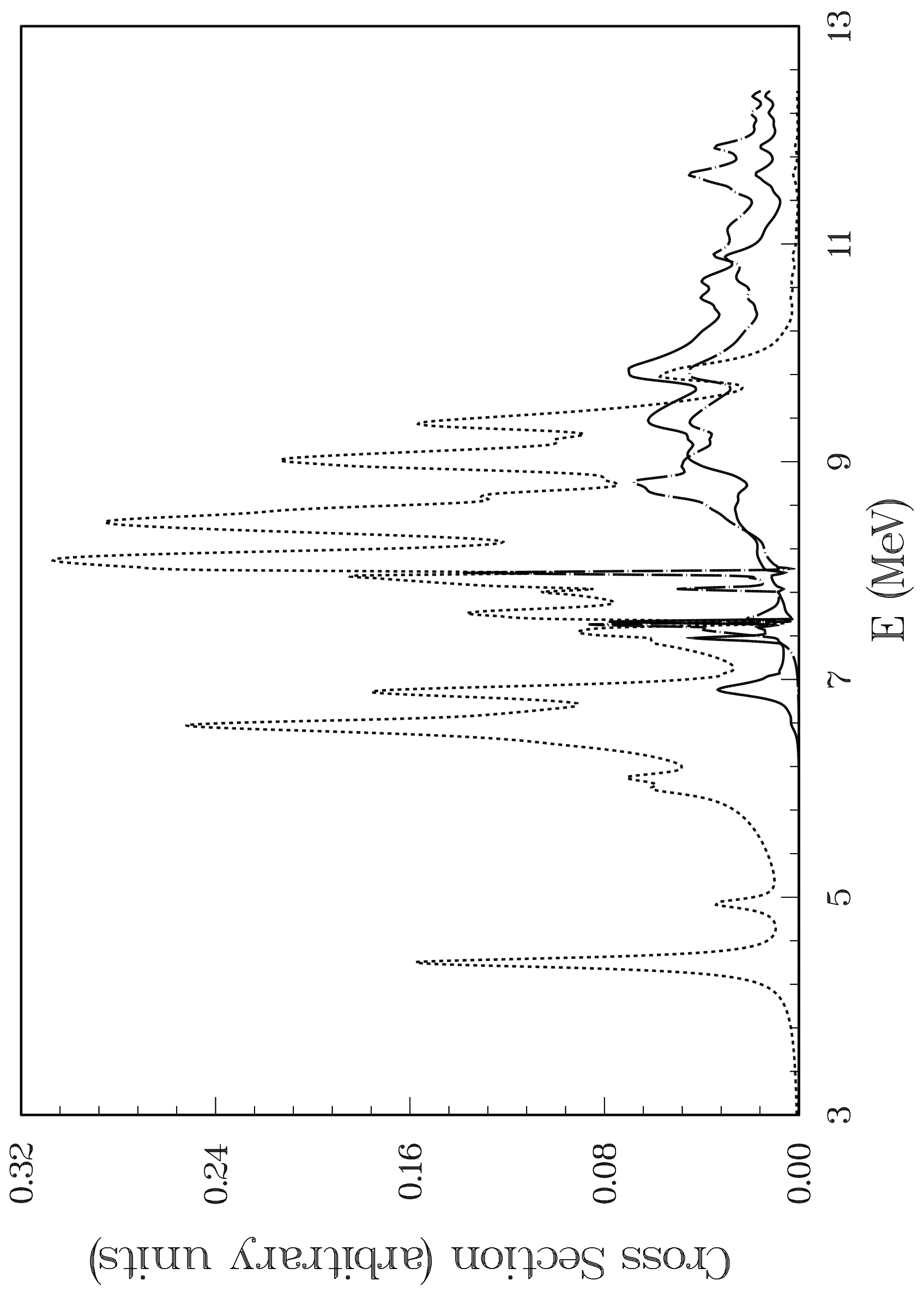

